\title{
Editorial: Advances and Challenges in Nanomedicine
}

\author{
Susan Hua ${ }^{1,2 *}$ and Sherry $Y . W u^{3}$ \\ ${ }^{1}$ Therapeutic Targeting Research Group, School of Biomedical Sciences and Pharmacy, University of Newcastle, Callaghan, \\ NSW, Australia, ${ }^{2}$ Hunter Medical Research Institute, New Lambton Heights, NSW, Australia, ${ }^{3}$ School of Biomedical \\ Sciences, University of Queensland, Brisbane, QLD, Australia
}

Keywords: nanomedicine, nanotechnology, nanoparticles, targeted drug delivery, translation

\section{Editorial on the Research Topic}

\section{Advances and Challenges in Nanomedicine}

The use of nanotechnology in medicine has the potential to have a significant impact on human health by improving the diagnosis, prevention and treatment of diseases. Nanomedicines typically encapsulate therapeutic and/or imaging compounds in submicrometer-sized carrier materials. In the last several decades, the application of nanomedicine for clinical purposes has received significant attention from academia, researchers, government, funding agencies, and regulatory bodies (Allen and Cullis, 2004; Sercombe et al., 2015; Hare et al., 2017; Hua et al.). Nanomedicines are generally intended to increase the therapeutic index of compounds by allowing more efficient delivery to the target site to enhance therapeutic efficacy and/or by minimizing accumulation in healthy body sites to reduce toxicity. Nanoencapsulation can also protect therapeutics from degradation in biological environments and can provide solubilization (Talekar et al., 2015; Mishra et al., 2017; Shajari et al., 2017). This e-Book focuses on articles that discuss the advances and challenges in the nanomedicine field across a broad range of topics. A brief summary of each article is provided below.

OPEN ACCESS

Edited and reviewed by:

Dominique J. Dubois,

Free University of Brussels, Belgium

*Correspondence: Susan Hua susan.hua@newcastle.edu.au

Specialty section: This article was submitted to Pharmaceutical Medicine and Outcomes Research, a section of the journal Frontiers in Pharmacology

Received: 28 October 2018 Accepted: 13 November 2018 Published: 29 November 2018

Citation: Hua S and Wu SY (2018) Editorial: Advances and Challenges in Nanomedicine.

Front. Pharmacol. 9:1397. doi: 10.3389/fphar.2018.01397
Nanomedicines can have a combination of chemical, physical, and biological properties that influences their in vivo behavior.

- Arms et al. addressed the principles and methodology of the available techniques for evaluating in vivo biodistribution of nanoparticles (Arms et al.). They further compared the advantages, limitations and capabilities of the techniques for assessing cellular, whole-organ and realtime accumulation (Arms et al.). Determining the in vivo biodistribution of nanoparticles following administration in animals and humans is an important component in the translational assessment of nanomedicines (Arms et al.).

- In addition, Robson et al. discussed the advantages and limitations of available imaging techniques used to evaluate the morphology of liposomal formulations (Robson et al.). Validating and controlling the morphology of nanoparticles is important for clinical translation, however it is generally difficult to control and not well-characterized (Robson et al.).

There are a number of factors that can impose significant obstacles to the clinical translation of nanomedicines, irrespective of whether they are therapeutically beneficial or not.

- Accomasso et al. summarized the current state regarding the safety evaluation of nano-based therapeutics and discussed the importance of risk assessment and risk minimization in the development of nanomedicines (Accomasso et al.). With the rapid growth in the use of nanomaterials for medical applications, the most urgent need is developing and validating novel and practical approaches that are able to determine potential short-term and long-term health risks, including the extrapolation of acute in vitro results for the prediction of chronic in vivo effects (Accomasso et al.). 
- More broadly, Hua et al. discussed the current trends and challenges in the clinical translation of nanoparticulate nanomedicines, as well as the potential pathways for translational development and commercialization (Hua et al.). Key issues related to the clinical development of nanoparticulate nanomedicines include biological challenges, biocompatibility and safety, large scale manufacturing, government regulations, intellectual property (IP), and overall cost-effectiveness in comparison to current therapies (Hua et al.).

Undoubtedly, the vast majority of nanomedicines in preclinical and clinical development as well as in clinical use are for targeting a wide variety of malignant tumors.

- Yang et al. reviewed the advances and challenges in utilizing exosomes for the delivery of cancer therapeutics (Yang and $\mathrm{Wu}$ ). Significant progress has been made to overcome major barriers for using exosomes as a delivery system, thereby opening a new promising avenue for cancer treatment (Yang and $\mathrm{Wu}$ ).

- Chang et al. highlighted the current understanding and challenges of biologically targeted magnetic hyperthermia to induce cancer cell death and potentially improve the effectiveness and safety of hyperthermia (Chang et al.). Application of an alternating magnetic field following administration of cancer-targeting magnetic nanoparticles that accumulate in the tumor allows preferential heating of malignant cancer cells (Chang et al.). Despite promising results in preclinical studies, there are a number of challenges that still need to be addressed before this technique can progress to the clinic (Chang et al.).

- Furthermore, Buss et al. discussed how nanotechnology can help overcome current obstacles for the treatment of bladder cancer. This includes how it can be used in non-muscle-invasive urothelial bladder cancer to facilitate combination chemotherapeutic and BCG (mycobacterium bovis bacillus Calmette-Guerin) immunotherapies (Buss et al.).

Modulation of the tumor microenvironment has recently emerged as an important strategy to improve the delivery of nanomedicines to tumors, given the importance of cancerassociated cells in tumor growth and metastasis.

- Zhang et al. discussed the existing approaches and strategies for modulating the tumor microenvironment to improve tumor perfusion (Zhang et al.). This enables accumulation of nanomedicines at the tumor site, facilitates extravasation of nanomedicines for improving transvascular transport, and enhances interstitial transport for optimizing the biodistribution of nanomedicines (Zhang et al.). These strategies may provide an opportunity for the development of novel combination chemotherapeutic regimens and reassessment of previously suboptimal compounds (Zhang et al.).

- Correspondingly, Harrison et al. addressed the advances and challenges of nucleic acid delivery of nanoparticles to the tumor microenvironment (Harrison et al.). Despite the development of various nanoparticle platforms to overcome nucleic acid delivery hurdles, several challenges still exist for effective tumor delivery (Harrison et al.). One such challenge has been the accumulation of nanoparticles in non-cancer cells within the tumor microenvironment, which has recently opened up novel therapeutic applications for nanoparticles (Harrison et al.).

The application of nanomedicine-based therapies for drug targeting to non-cancer conditions has also increased in recent years.

- Remiao et al. presented recent developments in nanotechnology to overcome impairments still faced by medically assisted reproductive technology (e.g. multiple pregnancy, ovary stimulation, and genetic disorders) and new perspectives for the further use of nanotechnology in reproductive medicine (Remião et al.). The application of nanotechnology approaches to reproductive medicine have provided strategies to improve diagnosis and increase specificity and sensitivity (Remião et al.).

- Fang et al. showed that liposome-encapsulated baicalein may have the potential to improve wound healing and restore skin structure after skin injury (Fang et al.). The study demonstrated that liposome-encapsulated baicalein can inhibit adipogenic differentiation medium (ADM)induced lipid accumulation and extracellular matrix formation in Hs68 fibroblasts through the suppression of lipogenesis enzymes and inflammatory responses (Fang et al.).

- In addition, Yan et al. have elucidated the possible pathways for layered double hydroxide (LDH) nanoparticles to enhance antigen (cross)-presentation on immune cells as adjuvants for protein vaccines (Yan et al.). Nanoparticles have been intensively investigated as adjuvants in new generation vaccines, however how these nanoparticles provoke immune responses is not well-understood (Yan et al.). This research would help to understand the nanoparticle adjuvant mechanism and further assist the design of new specific nanoparticles as more efficient nano-adjuvants (Yan et al.).

\section{SUMMARY}

Overall, nanomedicine has the potential to revolutionize the way we detect and treat damage to the human body. Although nanomedicines have demonstrated significant therapeutic advantages for a multitude of medical applications, their translation has not progressed as rapidly as the plethora of positive preclinical results would have suggested (Luxenhofer et al., 2014; Sercombe et al., 2015; Hare et al., 2017; Hua et al.). The experimental development of nanomedicines is continually progressing at a fast pace, however significant challenges still exist in promoting these platforms into clinically feasible therapies. Therefore, continued translational success will require communication and collaboration between experts involved in all stages of pharmaceutical development of nanotechnologies, 
including pharmaceutical design and manufacturing, cellular interactions and toxicology, as well as preclinical and clinical evaluation (Hua et al.).

\section{AUTHOR CONTRIBUTIONS}

SH drafted the manuscript. SH and SW critically reviewed the manuscript for important intellectual content.

\section{REFERENCES}

Allen, T. M., and Cullis, P. R. (2004). Drug delivery systems: entering the mainstream. Science 303, 1818-1822. doi: 10.1126/science.1095833

Hare, J. I., Lammers, T., Ashford, M. B., Puri, S., Storm, G., and Barry, S. T. (2017). Challenges and strategies in anti-cancer nanomedicine development: an industry perspective. Adv. Drug Deliv. Rev. 108, 25-38. doi: 10.1016/j.addr.2016.04.025

Luxenhofer, R., Barz, M., and Schillmeier, M. (2014). Quo vadis nanomedicine? Nanomedicine 9, 2083-2086. doi: 10.2217/ nnm. 14.140

Mishra, D. K., Balekar, N., and Mishra, P. K. (2017). Nanoengineered strategies for siRNA delivery: from target assessment to cancer therapeutic efficacy. Drug Deliv. Transl. Res. 7, 346-358. doi: 10.1007/ s13346-016-0352-5

Sercombe, L., Veerati, T., Moheimani, F., Wu, S. Y., Sood, A. K., and Hua, S. (2015). Advances and challenges of liposome assisted drug delivery. Front. Pharmacol. 6:286. doi: 10.3389/fphar.2015.00286

\section{ACKNOWLEDGMENTS}

We wish to thank The Pharmacy Research Trust of New South Wales, The Rebecca L. Cooper Medical Research Foundation, Gladys M. Brawn Fellowship, ausEE Research Foundation, and The University of Newcastle for providing financial support for our research.

Shajari, N., Mansoori, B., Davudian, S., Mohammadi, A., and Baradaran, B. (2017). Overcoming the challenges of siRNA delivery: nanoparticle strategies. Curr. Drug Deliv. 14, 36-46. doi: 10.2174/15672018136661608161 05408

Talekar, M., Tran, T. H., and Amiji, M. (2015). Translational nano-medicines: targeted therapeutic delivery for cancer and inflammatory diseases. AAPS J. 17, 813-827. doi: 10.1208/s12248-015-9772-2

Conflict of Interest Statement: The authors declare that the research was conducted in the absence of any commercial or financial relationships that could be construed as a potential conflict of interest.

Copyright (C) $2018 \mathrm{Hua}$ and $\mathrm{Wu}$. This is an open-access article distributed under the terms of the Creative Commons Attribution License (CC BY). The use, distribution or reproduction in other forums is permitted, provided the original author(s) and the copyright owner(s) are credited and that the original publication in this journal is cited, in accordance with accepted academic practice. No use, distribution or reproduction is permitted which does not comply with these terms. 